\title{
$\widehat{A}$ Madridge
}

madridge Journal of Behavioral and Social Sciences

Interconnecting Scientific World

Research Article

Open Access

\section{Rising prevalence of Osteopenia in young and middle aged Men in South Mumbai-An Alarming Situation}

\author{
Sharmin Bala ${ }^{\star}$ and Preeti Chhabria \\ Sir H N Reliance Foundation Hospital, Mumbai, India
}

\section{Article Info}

\author{
${ }^{*}$ Corresponding author: \\ Sharmin Bala \\ Flat no 15/B, 2nd Floor \\ New Buildings \\ Malcolm Baug \\ S.V Road, Jogeshwari west \\ Mumbai 400102 \\ India \\ Tel: +91-9967007953 \\ E-mail: sharminbala@gmail.com
}

Received: October 21, 2017

Accepted: November 20, 2017

Published: November 27, 2017

Citation: Bala S, Chhabria P. Rising prevalence of Osteopenia in young and middle aged Men in South Mumbai-An alarming situation. Madridge J Behav Soc Sci. 2017: 1(1): 31-35. doi: 10.18689/mjbss-1000105

Copyright: $\odot 2017$ The Author(s). This work is licensed under a Creative Commons Attribution 4.0 International License, which permits unrestricted use, distribution, and reproduction in any medium, provided the original work is properly cited.

Published by Madridge Publishers

\begin{abstract}
Background: Osteopenia is one of the common under-diagnosed medical conditions in males, with a prevalence of nearly $50 \%$ in India.
\end{abstract}

Objective: To analyse the prevalence and associations of Osteopenia in men in South Mumbai.

Design: Observational cross sectional study

Setting: A 345-bed, multi-specialty, tertiary care hospital and research centre.

Participants: 200 males visiting the 'Executive Health Check-up' department without the history of fractures were evaluated.

Measurements: The Bone Mineral Density of the subjects was measured on Dualenergy X-ray absorptiometry, based on the T-Spine and T-Hip values. The subjects were also evaluated clinically and biochemically.

Results: The prevalence of osteopenia was observed to be $55 \%$, and the prevalence of osteoporosis was $15.6 \%$. There is a significant positive correlation $(p<0.05)$ of T-Hip and Prolactin levels, and of T-Hip and TSH levels.

Conclusion: The alarming prevalence of osteopenia in young and middle-aged men in a metropolitan city in India is of great concern to the public health, which may in the future result in increased occurrence of osteoporosis, leading to greater burden on the health economy.

Keywords: Osteopenia; Men.

\section{Introduction}

Male osteoporosis is an important, although largely under-estimated public health problem. In both men and women, ageing is accompanied by continuous bone loss, followed by an exponential rise in the occurrence of osteoporotic fractures. Osteoporosis is a metabolic disorder of the bone, which is characterized by reduced bone density and micro-architectural deterioration, leading to increased bone fragility and susceptibility to fracture. Osteopenia is characterised by a generalized reduction in bone density, which is less severe than that in osteoporosis, usually caused by the resorption of bone at a rate which exceeds bone synthesis. Osteopenia is defined according to bone mineral density (BMD) assessment. According to the WHO classification of osteoporosis (Table 1) osteopenia is defined by bone densitometry as a T score -1 to -2.5 (A BMD value more than 1 standard deviation below the young adult mean, but less than 2 SDs below this value) [1]. 
Table 1. WHO osteoporosis classification

\begin{tabular}{|l|l|}
\hline \multicolumn{1}{|c|}{ Diagnosis } & T-score \\
\hline Normal & $>-1.0$ \\
\hline Osteopenia & $<-1.0,>-2.5$ \\
\hline Osteoporosis & $<-2.5$ \\
\hline Severe osteoporosis & $<-2.5$ plus fragility fractures \\
\hline
\end{tabular}

There are multiple causes for osteopenia, which generally include ageing, calcium and vitamin D deficiency, inactivity, and genetics [2]. Most of the published studies of osteopenia have documented the loss of BMD in women [3]. Of the few documented studies available, it has been found that osteopenia is one of the undetected medical conditions in Indian males, with a prevalence of almost $50 \%$ in the elderly males and $18 \%$ in young males $[4,5]$. Vertebral and hip fractures are linked to significant functional impairment and a greater risk of mortality in men than in women [6]. Low BMD is recognized as a major risk factor for fractures [7]. Osteoporosis in men accounts for more than $30-40 \%$ of overall fractures [8]. Medical evidence displays that approximately $80 \%$ of the urban Indian population is vitamin $D$ deficient, with hip fractures occurring about a decade earlier than in Western populations [9].

Dual-energy X-ray absorptiometry (DXA) is accepted as the reference method for measuring BMD at the spine and hip and to diagnose osteoporosis $[10,11]$. There is a paucity of research to assess the prevalence of Osteopenia in men. This study furnishes the reader with an estimate of the problem and the correlations of Osteopenia.

\section{Aim}

1. To analyse the prevalence of Osteopenia in men in Mumbai, India, by analysing the bone mineral density levels

2. Association of osteopenia with various biochemical parameters.

\section{Methods}

Two hundred males visiting the 'Executive Health Checkup' department were evaluated clinically (including anthropometry), and the biochemical values of Haemoglobin $(\mathrm{HB})$, serum Calcium (CA), Phosphorous (PHS), Uric acid (UA), Triglycerides, Low density Lipoprotein (LDL), High density Lipoprotein (HDL), Creatinine, Testosterone (TST), Prolactin (PLC), Thyroid stimulating hormone (TSH), Vitamin D (VTD), Vitamin B12 (VB12), Fasting blood sugar (FBS), Prostate specific antigen (PSA), and Alkaline Phosphatase (ALP) were measured.

The BMD was measured on 'Lunar iDXA' of GE Healthcare, utilizing the T-Spine and T-Hip values. The subjects were termed 'Osteopenic' if either the T scores of the spine or the hip were observed to be in the range -1 to -2.5 ; and 'Osteoporotic' if the T scores were less than or equal to -2.5 . The demographic and clinical data of the patients were retrieved from the hospital database applying the Systems, Applications and Products system (SAP).

Inclusion criteria: Men over 20 years old, who have completed the Full body DXA-BMD scan.
Exclusion criteria: Patients with medical disorders that are likely to affect BMD, those who had received steroids, antitubercular or anti-epileptic medication within the last one year, those who had fractures within the last two years.

History of smoking, alcohol consumption, history of osteoporosis or non-traumatic fractures in the family was retrieved for all subjects.

Data was entered in Microsoft Excel and analysed using Stata Version 13.1.The means and standard deviations, medians and inter quartile ranges (IQR) for the linear variables were calculated. We also calculated the proportions for the categorical variables, the Pearson Correlation Co-efficient to estimate the correlation between two linear variables. A p value of less than 0.05 was considered to be statistically significant.

\section{Results}

Of the 200 subjects evaluated according to the T-Spine score, 77 (38.7\%) were diagnosed with osteopenia, and 25 (12\%) with osteoporosis. 98 males (49.25\%) had normal T-scores of spine. According to the T-Hip score, 95 males (47.7\%) were diagnosed with osteopenia and 17 males (8.5\%) with osteoporosis. 88 males ( $44.5 \%$ ) had normal T-scores of hip (Table 2). In total, 108 men (55\%) were diagnosed with osteopenia and 32 men (16\%) were diagnosed with osteoporosis when T-Hip or T-Spine was evaluated. 60 individuals (30.15\%) had normal values of both T-Spine and T-Hip (Table 3). Prevalence of Osteoporosis was 15.6\% (95\% confidence: $10.8 \%-21.4 \%)$.

Table 2. Classification of subjects diagnosed as osteopenia and osteoporosis according to T-spine and T-Hip values

\begin{tabular}{|l|l|l|l|}
\hline T spine & Frequency & Percentage & Cumulative \\
\hline Normal & 98 & 49.25 & 49.25 \\
\hline Osteopenia & 77 & 38.69 & 87.94 \\
\hline Osteoporosis & 25 & 12.06 & 100 \\
\hline Total & 200 & 100 & \\
\hline T-Hip & Frequency & Percentage & Cumulative \\
\hline Normal & 88 & 44.22 & 44.22 \\
\hline Osteopenia & 95 & 47.74 & 91.96 \\
\hline Osteoporosis & 17 & 8.5 & 100 \\
\hline Total & 200 & 100 & \\
\hline
\end{tabular}

Table 3. Classification of subjects diagnosed as osteopenia and osteoporosis according to T-Spine or T-Hip values

\begin{tabular}{|l|l|l|l|}
\hline & Frequency & Percentage & Cumulative \\
\hline Normal & 60 & 30.15 & 30.15 \\
\hline Osteopenia & 108 & 54.27 & 84.42 \\
\hline Osteoporosis & 32 & 16 & 100 \\
\hline Total & 200 & 100 & \\
\hline
\end{tabular}

The subjects were divided according to age-groups (Table 4). Of the 25 subjects of less than 40 years of age, 16 (64\%) were diagnosed with osteopenia, and 2 (8\%) with osteoporosis. There were 45 subjects in the age-group of 40-49 years, of which 26 men (57.8\%) were identified with osteopenia, and 3 men (6.6\%) with osteoporosis. In the age-group of 50-59 years, of the 68 patients, 35 (51.5\%) were detected with osteopenia, and 10 (14.7\%) with osteoporosis. Of the 41 subjects in the age group of 60-69 years, 23 (56.1\%) were osteopenic and $10(24.4 \%)$ osteoporotic. Of the 19 patients aged over 70 years, $8(42.1 \%)$ were observed to be osteopenic, and $6(31.6 \%)$ osteoporotic. 
Table 4. Classification of data according to age of the subjects

\begin{tabular}{|l|l|l|l|}
\hline Age (years) & Normal & Osteopenia & Osteoporosis \\
\hline$<40$ & & & \\
\hline $\mathbf{n}$ & 7 & 16 & 2 \\
\hline$\%$ & 28 & 64 & 8 \\
\hline $40-49$ & & & \\
\hline $\mathbf{n}$ & 16 & 26 & 3 \\
\hline$\%$ & 35.5 & 57.8 & 6.6 \\
\hline $\mathbf{5 0 - 5 9}$ & & & \\
\hline $\mathbf{n}$ & 23 & 35 & 10 \\
\hline$\%$ & 33.8 & 51.47 & 14.7 \\
\hline $60-69$ & & & \\
\hline $\mathbf{n}$ & 8 & 23 & 10 \\
\hline$\%$ & 19.51 & 56.1 & 24.4 \\
\hline$>70$ & & & \\
\hline $\mathbf{n}$ & 5 & 8 & 6 \\
\hline$\%$ & 26.32 & 42.1 & 31.5 \\
\hline & & & \\
\hline Total (n) & 59 & 108 & 31 \\
\hline$\%$ & 29.8 & 54.5 & 15.6 \\
\hline
\end{tabular}

$\mathrm{n}=$ total number of individuals

Pearson $\mathrm{chi}^{2}(8)=11.9738$

$\operatorname{Pr}=0.152$

In the present study, 50\% subjects were regularly exercising, $37 \%$ had been diagnosed with a chronic disease (CD); $22 \%$ men were chronic smokers, 35\% were alcohol consumers (Table 5). The mean and SD of individual values has been illustrated in (Table 6). The median and interquartile range for Prolactin, Thyroid Stimulating Hormone (TSH), VTD, VB12, T-Spine, T-Hip is illustrated in (Table 7).

Table 5. Characteristics of the population

\begin{tabular}{|l|l|}
\hline & Percent \\
\hline Regular exercise & 50 \\
\hline Chronic disease & 37 \\
\hline Smokers & 22 \\
\hline Alcohol consumers & 35 \\
\hline
\end{tabular}

Table 6. The mean and standard deviation of individual values

\begin{tabular}{|l|l|l|l|l|l|}
\hline Variable & Observation & Mean & SD & Minimum & Maximum \\
\hline Age & 200 & 54.2 & 12 & 23 & 88 \\
\hline $\mathrm{Hb}$ & 200 & 14.1 & 1.2 & 8.9 & 16.8 \\
\hline Calcium & 196 & 9.4 & 0.4 & 8.6 & 10.4 \\
\hline Phosphorous & 200 & 3.5 & 0.6 & 2.1 & 7.8 \\
\hline Uric Acid & 200 & 5.8 & 1.2 & 2.9 & 8.3 \\
\hline Triglycerides & 200 & 143.2 & 80.2 & 34 & 526 \\
\hline LDL & 200 & 111.1 & 36.2 & 26 & 237 \\
\hline HDL & 200 & 45.4 & 11.4 & 24 & 110 \\
\hline Creatinine & 200 & 0.9 & 0.2 & 0.6 & 1.5 \\
\hline Testosterone & 145 & 428.3 & 132.7 & 53 & 857 \\
\hline FBS & 200 & 113.7 & 29.5 & 82 & 262 \\
\hline PSA & 196 & 1.2 & 1.2 & 0.2 & 8.8 \\
\hline ALP & 197 & 74 & 19.7 & 31 & 162 \\
\hline BMI & 200 & 27.2 & 5.07 & 18 & 71 \\
\hline
\end{tabular}

*Hb-Hemoglobin, LDL-Low density Lipoprotein, HDL-High density Lipoprotein, FBS-Fasting Blood Glucose, PSA-Prostate Specific Antigen, ALP-Alkaline Phosphatase, BMI-Basal Metabolic Index, SD-Standard Deviation

Table 7. Median and interquartile range for Prolactin, Thyroid Stimulating Hormone (TSH), Vitamin D, Vitamin B12, T-Spine, T-Hip

\begin{tabular}{|l|l|l|}
\hline Variable & Median value & Interquartile range \\
\hline Prolactin & 9.06 & 4 \\
\hline TSH & 2.5 & 1.89 \\
\hline vitamin D & 25.1 & 15.5 \\
\hline vitamin B12 & 394 & 332 \\
\hline T-Spine & -1 & 1.7 \\
\hline T-Hip & -1.1 & -1.2 \\
\hline
\end{tabular}

*TSH-Thyroid stimulating hormone

The data from Table 8 can be inferred as follows: There is a significant positive correlation $(\mathrm{SPC})(\mathrm{P}<0.05)$ of alcohol intake and $C D, S P C$ of TG and HB, SPC of TG and LDL, SPC of VTD and VB12, SPC of T-Hip and PLC, SPC of T-Hip and TSH, SPC of FBS and TG, SPC of PSA and age, SPC of ALP and HB, SPC of BMI and $C D, S P C$ of $B M I$ and $T G$; significant negative correlation (SNC) of HB and age, SNC of PHS and alcohol intake.

Table 8. Pearson Correlation Co-efficient table

\begin{tabular}{|c|c|c|c|c|c|c|c|c|c|c|c|c|c|c|c|c|c|c|c|c|c|c|c|}
\hline & AGE & SMK & ALC & EXR & $C D$ & HB & CA & PHS & UA & TG & LDL & HDL & TST & PLC & TSH & VTD & VTB12 & TSP & THP & FBS & PSA & ALP & BMI \\
\hline AGE & 1.000 & & & & & & & & & & & & & & & & & & & & & & \\
\hline SMK & -0.328 & 1.000 & & & & & & & & & & & & & & & & & & & & & \\
\hline ALC & -0.066 & 0.258 & 1.000 & & & & & & & & & & & & & & & & & & & & \\
\hline EXR & 0.004 & -0.290 & 0.171 & 1.000 & & & & & & & & & & & & & & & & & & & \\
\hline CD & 0.355 & -0.076 & 0.381 & -0.033 & 1.000 & & & & & & & & & & & & & & & & & & \\
\hline HB & -0.414 & -0.027 & -0.054 & -0.124 & -0.277 & 1.000 & & & & & & & & & & & & & & & & & \\
\hline CA & -0.219 & 0.246 & 0.004 & -0.400 & -0.058 & 0.247 & 1.000 & & & & & & & & & & & & & & & & \\
\hline PHS & -0.058 & -0.006 & -0.413 & -1.000 & -0.169 & 0.066 & 0.237 & 1.000 & & & & & & & & & & & & & & & \\
\hline UA & 0.085 & 0.278 & -0.004 & -0.033 & 0.091 & -0.103 & 0.129 & 0.004 & 1.000 & & & & & & & & & & & & & & \\
\hline TG & -0.056 & -0.191 & -0.025 & 0.103 & -0.095 & 0.531 & 0.069 & 0.288 & 0.025 & 1.000 & & & & & & & & & & & & & \\
\hline LDL & -0.138 & -0.169 & -0.095 & 0.211 & -0.268 & 30.244 & 0.254 & 0.119 & 0.198 & 0.500 & 1.000 & & & & & & & & & & & & \\
\hline $\mathrm{HDL}$ & 0.123 & 0.317 & 0.235 & -0.008 & 0.050 & -0.120 & 0.094 & 0.198 & -0.353 & -0.322 & -0.257 & 1.000 & & & & & & & & & & & \\
\hline TST & -0.193 & -0.186 & -0.041 & 0.050 & -0.231 & 0.287 & -0.001 & 0.010 & -0.165 & 0.188 & 0.206 & $|-0.159|$ & 1.000 & & & & & & & & & & \\
\hline PLC & 0.118 & -0.147 & 0.066 & 0.173 & 0.243 & 0.136 & 0.076 & 0.148 & -0.047 & 0.285 & 0.177 & 0.164 & -0.033 & 1.000 & & & & & & & & & \\
\hline TSH & 0.193 & -0.262 & -0.285 & 0.037 & -0.087 & 0.050 & -0.138 & 0.179 & 0.049 & 0.263 & 0.005 & -0.052 & 0.109 & 0.288 & 1.000 & & & & & & & & \\
\hline VTD & 0.086 & 0.006 & 0.264 & 0.017 & 0.116 & 0.057 & 0.353 & 0.060 & 0.003 & 0.031 & -0.020 & 0.070 & 0.055 & 0.005 & -0.186 & 1.000 & & & & & & & \\
\hline VTB12 & 0.062 & 0.191 & 0.066 & -0.037 & 0.029 & 0.177 & 0.136 & 0.292 & -0.060 & 0.092 & -0.308 & 0.277 & 0.049 & -0.003 & -0.020 & 0.515 & 1.000 & & & & & & \\
\hline TSP & 0.049 & 0.082 & 0.153 & -0.033 & 0.277 & 0.045 & -0.104 & -0.060 & 0.060 & -0.057 & -0.157 & -0.024 & 0.073 & 0.351 & 0.285 & 0.126 & 0.295 & 1.000 & & & & & \\
\hline THP & 0.025 & 0.044 & 0.116 & -0.186 & 0.116 & 0.126 & -0.051 & -0.172 & 0.166 & 0.074 & -0.101 & -0.244 & 0.168 & 0.416 & 0.380 & 0.090 & 0.133 & 0.739 & 1.000 & & & & \\
\hline FBS & 0.300 & -0.202 & -0.240 & -0.240 & 0.099 & 0.218 & 0.117 & 0.185 & 0.066 & 0.364 & 0.084 & -0.232 & -0.060 & 0.127 & 0.156 & -0.104 & -0.056 & 0.181 & 0.029 & 1.000 & & & \\
\hline PSA & 0.477 & -0.180 & 0.256 & 0.243 & 0.342 & -0.171 & -0.154 & -0.258 & 0.131 & -0.049 & 0.144 & 0.104 & 0.348 & 0.188 & 0.163 & 0.148 & -0.124 & 0.139 & 0.109 & -0.013 & 1.000 & & \\
\hline ALP & 0.003 & -0.333 & -0.157 & 0.124 & -0.033 & 0.377 & 0.044 & 0.077 & 0.044 & 0.205 & 0.359 & -0.179 & 0.153 & 0.046 & -0.037 & -0.083 & -0.276 & -0.321 & -0.277 & 0.243 & 0.088 & 1.000 & \\
\hline BMI & 0.350 & -0.208 & 0.074 & -0.174 & 0.385 & 0.005 & -0.182 & -0.058 & 0.086 & 0.390 & 0.061 & -0.191 & -0.014 & 0.352 & 0.205 & 0.284 & 0.112 & 0.265 & 0.330 & 0.294 & 0.285 & -0.064 & 1.000 \\
\hline
\end{tabular}

*SMK-Smokers, ALC- Alcohol consumers, EXR- Exercise, CD- Chronic disease, HB-Haemoglobin, CA-Calcium, PHS-Phosphorous, UA-Uric Acid, TG-Triglycerides, LDL-Low density lipoprotein, HDL-High density lipoprotein, TST-Testosterone, PLC-Prolactin, TSH-Thyroid stimulating hormone, VTD-Vitamin D, VTB12-Vitamin B12, TSP- T Spine, THP-T-Hip, FBS-Fasting blood glucose, PSA-Prostate specific antigen, ALP-Alkaline phosphatase, BMI-Body mass index 


\section{Discussion}

It is evident from the literature that one of five men and every $2^{\text {nd }}$ woman are at a risk of osteoporotic fractures after the age of 50 years [12]. Astonishingly, we have observed a high prevalence of osteopenia in males younger than 50 years, which is a novel finding. A detailed study of osteopenia has revealed that almost half of the causes are potentially treatable [8]. The prevalence of Osteopenia and Osteoporosis in South India (58\% and $20 \%$ respectively) and in Mumbai (59.7\% and 18.3\%) conducted earlier, are similar to the findings of the current study ( $55 \%$ and $16 \%$ respectively). $(5,8)$ The biochemical values of the subjects in the present study were in the normal Laboratory Reference Ranges in Healthy Adults [13]. The average LDL and $\mathrm{BMI}$ values were found to be marginally higher than the normal range. VTD values were lower than the reference range.

BMD is an important risk factor for fracture and is similar in both sexes [14]. In our study, it is observed that as the patient's age, the probability of osteopenia and osteoporosis increases, which is similar to the findings in a recent study conducted in India and USA [8, 15]. Majority of the chronic disorders observed among the patients were diabetes mellitus, cardiovascular diseases and mental health illness. Studies have concluded that alcohol consumption is one of the major avoidable risk factors for the occurrence of chronic diseases, similar to the findings of our study [16]. It was observed that in the ageing population HB values decrease, and there is a higher incidence of Anaemia; the finding is similar to a recent study conducted in Germany [17]. Our study demonstrates that with increase in the intake of alcohol, blood Phosphorous levels decrease, which is similar to the study by Paolo et al [18].

A few novel observations were found in our study, which are not published elsewhere. Vitamin B12 and Vitamin D levels are directly proportional to each other. TG levels and LDL levels increase proportionately. Low PLC and TSH levels were observed to be moderately associated with Osteopenia. In contrast to our findings, Ajmal et al observed that hyperprolactinemia-induced oestrogen deficiency, in place of PLC itself, is the major factor in the development of osteopenia Hyperprolactinemia often occurs in patients receiving medications for psychiatric disorders. Patients with drug-induced hyperprolactinemia should be managed expectantly with attention to the risks of osteoporosis [19]. Howes et al has observed that BMD values are not clinically correlated with PLC levels in patients taking antipsychotic treatment [20]. In contrast to women, most males do not develop overt hypogonadism with ageing. Thus, bone loss proceeds slowly in middle-aged men. Male osteoporosis has more heterogeneous factors with multiple underlying causes in any given individual [21]. Further research is required to assess the correlation of lower prolactin levels in men and the development of osteopenia.

The conclusion of the study of Lee et al and Svare et al is comparable to the finding of the present study; BMD is directly proportional to TSH levels [22, 23]. The findings of a similar study conducted in USA suggest that a low normal TSH level in men is associated with a lower bone mineral density in men; which is similar to our study [24]. Thyroid hormone is essential for normal growth and bone development. Thyroid hormone deficiency results in delayed skeletal development, and growth arrest accompanied by epiphyseal dysgenesis. Thyroid hormone accelerates bone turnover and bone loss, and has a greater effect on cortical bone than on trabecular bone. The bone mineralization is reduced and the rate of bone turnover is increased in thyrotoxicosis, leading to an increased risk of hip fractures [22]. Hyperthyroidism in adulthood is associated with increased bone turnover and a reduction in BMD at various skeletal sites due to increased cortical porosity and accelerated bone loss. Population studies haveshownthat both hypothyroidismand hyperthyroidism in adults may be associated with an increased fracture risk [25].

FBS levels and TG levels increase proportionately, which is comparable to the findings of a study conducted in Canada [26]. With increase in BMI, TGs increase and there is an increased incidence of chronic diseases [27]. The PSA levels have shown to increase proportionately with the age of the subjects, which has also been reported in a population study in China [28]. There is no significant correlation of occurrence of osteopenia with age, smoking, exercise, alcohol intake, presence of chronic diseases, HB levels, CA, PHS, UA, TG, LDL, HDL, TST, VTD, VB12, FBS, PSA, $A L P, B M I$. In a recent study it was observed that haemoglobin levels and anaemia are negatively and independently associated with bone mass and density [29]. A study in the elderly population established that CA and VTD supplementation leads to a moderate reduction in bone loss [30]. Nabipour et al observed that higher serum UA levels are associated with greater BMD at all skeletal sites, and with a lesser prevalence of vertebral and non-vertebral fractures in older men [31]. A research study in USA concluded that cholesterol levels in men and women from young adulthood to middle age years cannot predict subsequent bone mineral density in elderly years [32]. Snyder at al observed that increasing the serum testosterone concentrations of normal men over 65 years of age did not increase lumbar spine bone density overall, but did increase it in those men with low pre-treatment serum testosterone concentrations [33]. Vitamin B12 deficiency may be an important modifiable risk factor for osteoporosis [34]. In the Rotterdam study, it was observed that higher blood glucose levels leading to diabetes increases the risk of osteoporosis [35]. Conde et al discovered that Prostate-specific antigen and higher BMI was an independent predictor of low BMD [15].

\section{Conclusion}

There is a high detection rate of unnoticed osteopenia in young and middle aged males, besides the high prevalence in elderly males. The prevalence rates of Osteopenia have been corroborated with the other studies conducted in India. Low TSH and Prolactin levels are associated with Osteopenia; however, more studies are needed to highlight the associations of Osteopenia. In India, the screening for osteopenia in males at an earlier age is necessary.

\section{Acknowledgment}

We are grateful to Dr. Maninder Setia for assisting with the Statitistical Analysis for the study. 
Statement of contributions of authors: Study conception and design: Dr. Preeti Chhabria, Acquisition of data: Dr. Sharmin Bala, Analysis and interpretation of data: Dr. Sharmin Bala, Drafting of manuscript: Dr. Sharmin Bala, Critical revision: Dr. Preeti Chhabria.

Compliance with Ethical Standards: Statement of human rights Ethical approval: All procedures performed in studies involving human participants were in accordance with the ethical standards of the institutional and/or national research committee and with the 1964 Helsinki declaration and its later amendments or comparable ethical standards.

Disclosure of potential conflicts of interest: The authors declare no conflict of interest.

Research involving Human Participants: For this type of study formal consent is not required, since complete anonymity is maintained.

Funding: No funding provided.

\section{References}

1. Binkley N. Osteoporosis in men. Arq Bras Endocrinol Metabol. 2006; 50(4): 764-74.

2. Karaguzel G, Holick MF. Diagnosis and treatment of osteopenia. Reviews in Endocrine and Metabolic Disorders. 2010; 11(4): 237-51. doi: 10.1007/ s11154-010-9154-0

3. Adler RA. Osteoporosis in men: a review. Bone Res. 2014; 2. doi: 10.1038/ boneres.2014.1

4. Agrawal NK, Sharma B. Prevalence of osteoporosis in otherwise healthy Indian males aged 50 years and above. Archives of osteoporosis. 2013; 8: 116. doi: $10.1155 / 2014 / 723238$

5. Multani SK, Sarathi V, Shivane V, et al. Study of bone mineral density in resident doctors working at a teaching hospital. J Postgrad Med. 2010; 56(2): 65-70. doi: 10.4103/0022-3859.65272

6. Berry SD, Miller R. Falls: Epidemiology, Pathophysiology, and Relationship to Fracture. Current osteoporosis reports. 2008; 6(4): 149-54. doi: 10.1007/ s11914-008-0026-4

7. Pisani $P$, Renna MD, Conversano $F$, et al. Major osteoporotic fragility fractures: Risk factor updates and societal impact. World Journal of Orthopedics. 2016; 7(3): 171-81. doi: 10.5312/wjo.v7.i3.171

8. Shetty $S$, Kapoor N, Naik D, et al. Osteoporosis in Healthy South Indian Males and the Influence of Life Style Factors and Vitamin D Status on Bone Mineral Density. Journal of Osteoporosis. 2014; 2014: 5. doi: 10.1155/2014/723238

9. Mithal A, Bansal B, Kyer CS, Ebeling P. The Asia-Pacific Regional AuditEpidemiology, Costs, and Burden of Osteoporosis in India 2013: A report of International Osteoporosis Foundation. Indian J Endocrinol Metab. 2014; 18(4): 449-54. doi: 10.4103/2230-8210.137485

10. El Maghraoui A, Roux C. DXA scanning in clinical practice. QJM. 2008; 101(8): 605-17. doi: 10.1093/qjmed/hcn022

11. Afzelius P, Garding MM, Molsted S. Dual-energy X-ray absorptiometry of both hips helps appropriate diagnosis of low bone mineral density and osteoporosis. Diagnostics. 2017; 7(3). doi: 10.3390/diagnostics7030041.

12. Tuck SP, Datta HK. Osteoporosis in the aging male: Treatment options. Clinical Interventions in Aging. 2007; 2(4): 521-36. doi: 10.2147/CIA.S820

13. Farinde A. Lab Values, Normal Adult. Drugs and Diseases. 2014.

14. Johnell O, Kanis JA, Oden A, et al. Predictive value of BMD for hip and other fractures. J Bone Miner Res. 2005; 20(7): 1185-94. doi: 10.1359/ JBMR.050304

15. Conde FA, Sarna L, Oka RK, Vredevoe DL, Rettig MB, Aronson WJ. Age, body mass index, and serum prostate-specific antigen correlate with bone loss in men with prostate cancer not receiving androgen deprivation therapy. Urology. 2004; 64(2): 335-40. doi: 10.1016/j.urology.2004.03.036
16. Rehm J, Mathers C, Popova S, et al. Global burden of disease and injury and economic cost attributable to alcohol use and alcohol-use disorders. Lancet. 2009; 373(9682): 2223-33. doi: 10.1016/S0140-6736(09)60746-7

17. Röhrig $G$, Gütgemann I, von Gersdorff $G$, et al. Aktuelle Einblicke in die Anämie im Alter. Z Gerontol Geriatr. 2017; 1-5. doi: 10.1007/s00391-017$1212-8$

18. Angeli $P$, Gatta $A$, Caregaro $L$, et al. Hypophosphatemia and renal tubular dysfunction in alcoholics: Are they related to liver function impairment? Gastroenterology. 1991; 100(2): 502-12. doi: 10.1016/0016-5085(91)90223-8

19. Ajmal A, Joffe $H$, Nachtigall LB. Psychotropic-Induced Hyperprolactinemia: A Clinical Review. Psychosomatics. 2014; 55(1): 29-36. doi: 10.1016/j. psym.2013.08.008

20. Howes OD, Wheeler MJ, Meaney AM, et al. Bone mineral density and its relationship to prolactin levels in patients taking antipsychotic treatment. J Clin Psychopharmacol. 2005; 25(3): 259-61.

21. Kim BJ, Lee SH, Bae SJ, et al. The association between serum thyrotropin (TSH) levels and bone mineral density in healthy euthyroid men. Clin Endocrinol (Oxf). 2010; 73(3): 396-403. doi: 10.1111/j.1365-2265.2010.03818.x

22. Lee SJ, Kim KM, Lee EY, et al. Low Normal TSH levels are Associated with Impaired BMD and Hip Geometry in the Elderly. Aging Dis. 2016; 7(6): 734-43. doi: 10.14336/AD.2016.0325

23. Svare A, Nilsen TI, Bjoro T, et al. Hyperthyroid levels of TSH correlate with low bone mineral density: the HUNT 2 study. Eur J Endocrinol. 2009; 161(5): 779-86. doi: 10.1530/EJE-09-0139

24. THYROID AND BONE: Thyroid Hormone Levels and Bone Density in Men. 2010.

25. Roef G, Lapauw B, Goemaere $\mathrm{S}$, et al. Thyroid hormone status within the euthyroid range is associated with bone mass and density in healthy young men at the age of peak bone mass. Bone. 2011; 48(2).

26. Riediger ND, Clark K, Lukianchuk V, Roulette J, Bruce $S$. Fasting triglycerides as a predictor of incident diabetes, insulin resistance and $\beta$-cell function in a Canadian First Nation. Int J Circumpolar Health. 2017; 76(1). doi: 10.1080/22423982.2017.1310444

27. Bray GA. Medical Consequences of Obesity. The Journal of Clinical Endocrinology \& Metabolism. 2004; 89(6): 2583-9. doi: 10.1210/jc.2004-0535

28. Xiao LH, Chen PR, Gou ZP, et al. Prostate cancer prediction using the random forest algorithm that takes into account transrectal ultrasound findings, age, and serum levels of prostate-specific antigen. Asian Journal of Andrology. 2017; 19(5): 586-90. doi: 10.4103/1008-682X.186884

29. Cesari $M$, Pahor $M$, Lauretani $F$, et al. Bone density and hemoglobin levels in older persons: results from the InCHIANTI study. Osteoporos Int. 2005; 16(6): 691-9. doi: 10.1007/s00198-004-1739-6

30. Dawson-Hughes B, Harris SS, Krall EA, Dallal GE. Effect of Calcium and Vitamin D Supplementation on Bone Density in Men and Women 65 Years of Age or Older. N Engl J Med. 1997; 337(10): 670-6. doi: 10.1056/ NEJM199709043371003

31. Nabipour I, Sambrook PN, Blyth FM, et al. Serum uric acid is associated with bone health in older men: A cross-sectional population-based study. J Bone Miner Res. 2011; 26(5): 955-64. doi: 10.1002/jbmr.286

32. Samelson EJ, Cupples LA, Hannan MT, et al. Long-term effects of serum cholesterol on bone mineral density in women and men: the Framingham Osteoporosis Study. Bone. 2004; 34(3): 557-61. doi: 10.1016/j. bone.2003.11.024

33. Snyder PJ, Peachey $\mathrm{H}$, Hannoush $\mathrm{P}$, et al. Effect of Testosterone Treatment on Bone Mineral Density in Men Over 65 Years of Age. The Journal of Clinical Endocrinology \& Metabolism. 1999; 84(6): 1966-72. doi: 10.1210/ jcem.84.6.5741

34. Tucker KL, Hannan MT, Qiao N, et al. Low Plasma Vitamin B12 Is Associated With Lower BMD: The Framingham Osteoporosis Study. J Bone Miner Res. 2005; 20(1): 152-8. doi: 10.1359/JBMR.041018

35. de Liefde II, van der Klift $\mathrm{M}$, de Laet $\mathrm{CE}$, et al. Bone mineral density and fracture risk in type-2 diabetes mellitus: the Rotterdam Study. Osteoporos Int. 2005; 16(12): 1713-20. doi: 10.1007/s00198-005-1909-1 